\title{
Ppp Project's Risk Management and Countermeasures
}

\author{
YangChenhui \\ SiChuan transportation vocational technical college 611130
}

Keywords: Ppp project, risk management , life cycle ,risk factors, model ,measures

\begin{abstract}
Ppp project is a financing model in this new era, relieving pressure of the government to improve the service quality and operational efficiency of project results, while providing strategic choice for the relevant financial institutions.Ppp project's life cycle is longer, the external presence of various objective reasons has a certain influence on execution of the project, so the project in addition to the general project has risks, since the extension itself on time and space, the external environment and internal factors influence is more complex, the risk was higher than others. How to prevent Ppp project risk effectively is an urgent problem. In this paper, the first we analysis of PPP project risk at home and abroad,and on the basis of the PPP project risk control model was constructed to analyze the influence factors of the PPP project risk ,so we can put forward the reasonable measures.

Ppp project refers to a pattern of funding public infrastructure projects, in which the private partners for specific industries and enterprises in the consortium, the state-owned companies and the private sector, the public partner is the national or local government. Ppp public services in order to solve the lack of funding, poor infrastructure and the level of capital efficiency and other issues, the State Ppp first application of the UK, China as a large developing country, the introduction of Ppp financing model is imperative. First, the construction of public infrastructure investment is very large, the government's financial allocation followed by far not meet the investment needs of the rapid development of private economy for our final application Ppp financing model has created favorable conditions. After Chinese accession into the WTO and the investment market,the financing model is also more in line with Chinese national conditions require. Long-term public financing risk management project countries accustomed to risk-taking and an additional investment, public investment in the financing process, only focusing on short-term interests, ignoring the risk of the project. Must enhance the risk management capability of public projects for this situation, establish a risk management mechanism.

Current domestic Ppp project finance is still in its infancy, fewer instances involved and countries of Ppp financing project studies were combined with their national conditions, Chinese national conditions are a huge difference with other countries, it is not on track to borrow his experience in the country. Ppp financing of the project risk analysis, the results should be in line with Chinese actual situation,build domestic situation of risk control model,and a reasonable response measures.
\end{abstract}

\section{Ppp project's theoretical basis}

Ppp is a means for the government to finance public projects, and want to find out about Ppp financing, we must understand Ppp clients. Athlete foreign general government public programs called "government project", there are some countries referring to as "public works." Government public programs in order to adapt to the country's economic development, to meet the social life and cultural needs, through the issuance of government bonds, financial investment and the way of local financial vouchers for financing. Public goods is an article, should be emphasized that public goods non-competitive and non-exclusive.Related studies show that there were a lot of public goods and private goods nature of the substance is between, public goods may be subject to public goods division, pure public goods and private goods according to the attributes of public goods. In real life, the real pure public goods are rare, most of the items are quasi-public goods. And quasi-public goods can also be divided into club-type public goods and crowded type of quasi-public 
goods.Club-type quasi-public goods charge by way of use,the user has a non-competitive in the article, for example: cable TV, more than the increase does not affect the use of personnel will effect others.Consumption crowded type of quasi-public goods with a non-exclusive features, when the number reaches a certain limit consumer products will appear crowded, such as roads do not charge fees when the vehicle reaches a certain limit will restrict the use by others of the road .

\section{Foreign Ppp project risk current situation}

In economics, as well as other academic fields "risk" does not exist any technical content, the real meaning of risk is the possibility of damages. Project risk management thinking earliest England Professor CB Chapman raised its emphasis on large-scale high-level risk management have been studied and proposed various kinds of risk analysis techniques to integrate ideas,thereby helping Project management. Abroad Ppp project financing risk, the risk into the macro micro and medium three types. The risks of macro type usually associated with the social environment, economic environment, legal environment, and political environment there is a close relationship; microscopic risk is due to the contract management of the private sector, government agencies due to the different own characteristics, and therefore in the procurement process the presence of shareholder relations formed by the risk of this type of risk a detailed classification; the medium type of risk refers primarily to the completion of the item in question. Ppp's common in project risk study are the following:

A, Under effective competition in the market, to maximize the advantages of private capital investors and government resources for effective combination to achieve the best results of public services, public construction.

B,For the whole process of the risk of public projects, there are some differences in different countries on the mode selected, the UK emphasized that the privatization, France attention is between the private and public sectors to find a balance, emphasis is to get the government direct management.

C,Against Ppp project finance management, with particular emphasis on foreign quantitative analysis of risk factors for project financing division, identify systematic research project research methods also have a very deep understanding. At present the combination of Internet technology, computer technology for different phases of the project risks are identified, it is possible to make more effective risk management measures.

\section{The domestic research status Ppp project risk}

After Ppp project introduces domestic experts and scholars of our country also Ppp project risk indicators for a certain amount of research on Ppp financing projects in the risk classification, LaiGuojin States and other scholars Ppp project financing risks are divided into operational risk, financial risk, technical risk, currency risk and policy risk; and KeYongjian and other students will be divided Ppp financing risk for financial risks, market return risk, legal risk, operational risk, construction risk, and political risk. Some scholars of the project by analyzing the characteristics of the analysis process, from the overall design framework Ppp project risk systems and composition are described, and gives the reasoning mechanism based instance. Some scholars also from the perspective of different participants of the project risk factors faced by the respective analyzes, first in handicap between private investors and the government to share more reasonable. In short, Ppp project financing project risk analysis, foreign countries have in theory more in-depth research, the findings are more specific, but the theory of domestic Ppp Financing projects still in the initial period, the theoretical basis is weak.

\section{Ppp project risk analysis}

In order to study the progress and rule of Ppp project financing risks more clearly understood to Articles, databases and other literature sources,use the advanced search retrieved a total way of 
project financing analysis about Ppp ask you to supply 80 parts, documents above statistical analysis showed that the risk of research literature about Ppp project appeared in 2002, after China, the relevant literature after 2004 gradually increased in recent years with the promotion Ppp mode applications, which is getting attention higher. Current research Ppp financing risk in the peak period of development, especially in the past two years with the Ppp mode in infrastructure projects are more and more wide, the corresponding research results are also more and more. Currently in qualitative analysis to quantitative analysis, also we made some research, risk analysis Ppp financing projects with mufti-angle, mufti-level features.

Ppp items exist in the countries in which the application stage some differences, countries Ppp project understanding and awareness vary, depending on the operating conditions of different infrastructure projects Ppp project can be divided as follows: class outsourcing, franchising category and class of privatization. Ppp outsourcing project where the class, which is attributed to the ownership of the Government, the private is carried out by way of contract to the castle several tasks throughout the project or task. In Ppp outsourcing class, private sector projects undertaken relatively low risk. Ppp outsourcing class also includes two services outsourcing and outsourcing management. Franchise type Ppp project is a common risk Ppp project through cooperation mechanisms with the public sector, revenue is shared. In order for the private sector and a more balanced public service, the public sector will be given the franchise based on the actual earnings of the project company or certain compensation charge a management fee. In this type of project management Ppp government will affect the operation of the franchise projects to a large extent. But on the other hand, under the supervision of government departments, can save the cost of the project to some extent, improve service quality of the project. Ppp for privatization projects, the private sector is responsible for all investment projects under the supervision of the government's charge to the user, to profit investments. Since the type of ownership of the project Ppp always are private, and there is limited recourse features, so the private commitment in this type of project, project Ppp greatest risk. In general, the existing public programs for the expansion, will choose WA, BBO, LBO to run the model, if the formation of a new public projects choose BOOT, BOT, BTO and other operating modes.All in all, the application operation mode, select the operating mode should be used according to the actual situation, in order to ensure quality while improving efficiency.

\section{Construction Ppp project risk control model}

The nature and importance of the presence of the different stages of the project Ppp risk of some other car,the exposure to each phase of the project will be able to point out the orderly arrangement of risk management focus, and promote risk management efficiency.So we build the risk control model of the following:

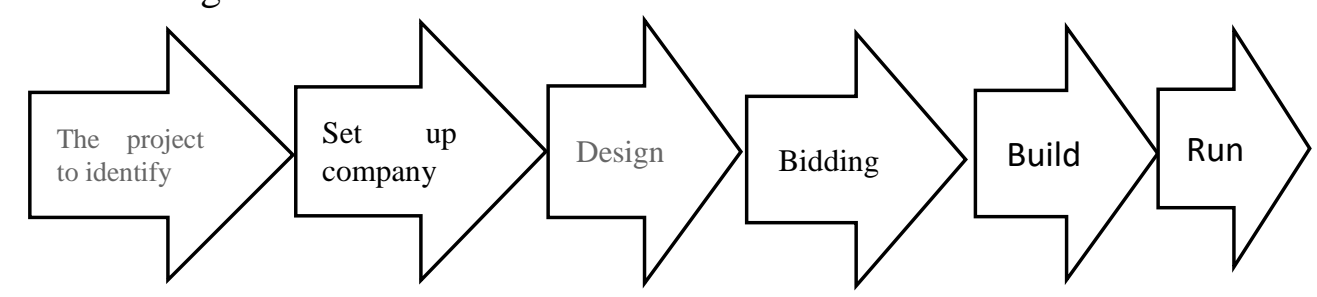

Each stage of the risk for importance 

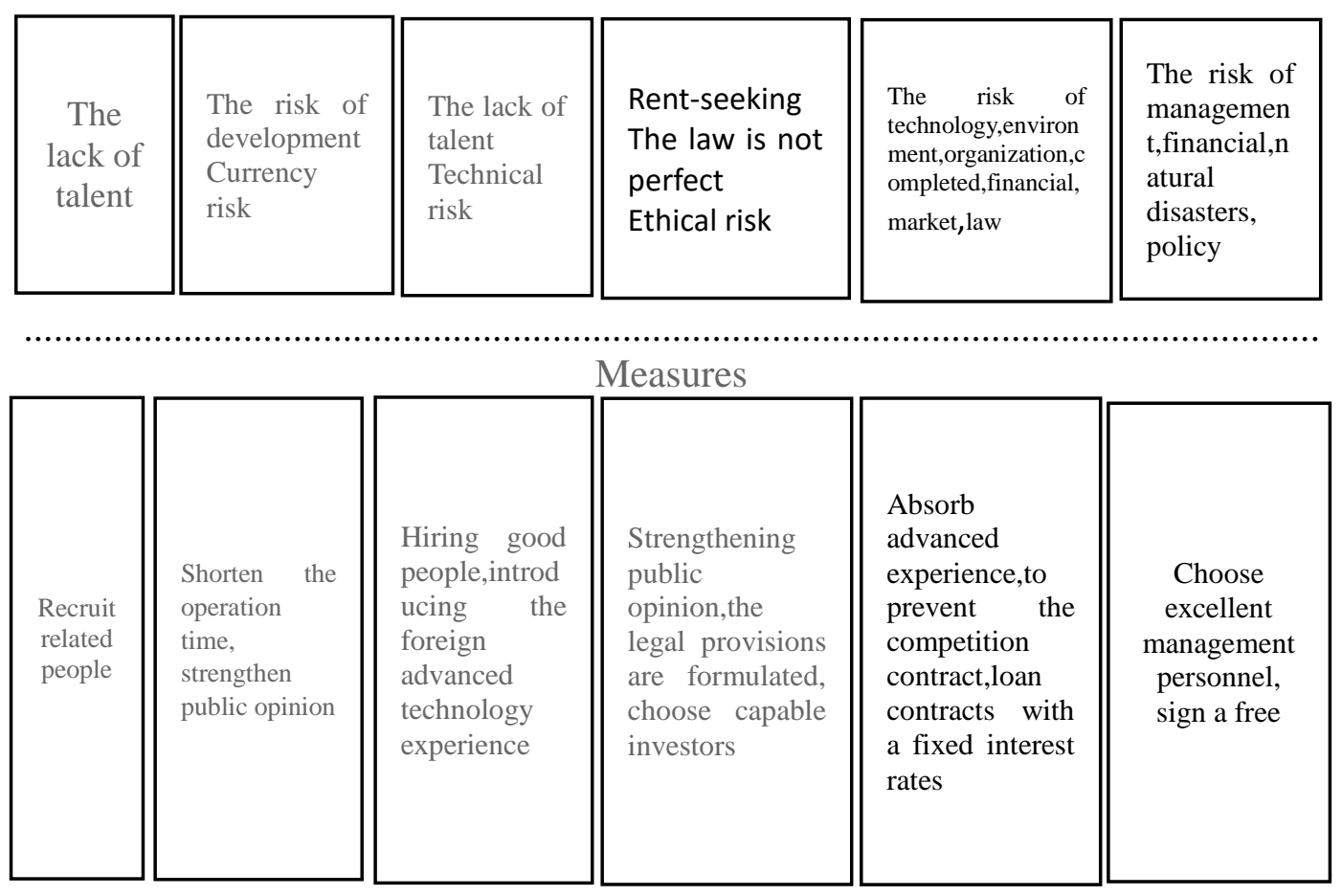

The Ppp project risk control function model

\section{Ppp project risk response measures}

Ppp project risk response measures to contain the risk of avoidance, retention,and transfer of control.

Risk avoidance:risk aversion is behind the project risk assessment,if the probability of risk is higher, the larger the losses caused, and no other countermeasures so that risk reduction should be chosen to abandon the project,so as to effectively reduce the loss occurred. Use of risk avoidance measures in the face of catastrophic risk is the most effective, but to abandon the project also means giving up certain probability.

Risk retention: Risk retention is a project risk management remain in the interior, through the internal control measures to reduce the risk, you can not take any action against these risks retained. Compared with other risk measures and does not change the objective nature of the risk.

Risk Control: Risk control is positive, proactive measures to deal with the risk, risk control efforts can be divided into two loss reduction and loss prevention aspects. In general, the risk of loss prevention and loss control room to reduce two kinds of organic integration.

Transfer of risk: risk management, risk transfer is a very important response measures can not be effective and avoid some of the risk in the transfer itself is very effective when the risks can not afford to choose risk. It should be noted that the risk transfer does not eliminate risk, but will be transferred in the interests of risk management that can be obtained to others, the project managers will not face the risk has been transferred.

In addition to the above measures unexpected, due to the weight of public administration regulatory approval is more serious light, in order to effectively play the advantages of life-cycle mode Ppp institutional mechanisms, so Ppp project should be value for money through quantitative assessment:

Identification phase: the identification phase of the project, because this time the information is not comprehensive enough, it should be mainly qualitative assessment, combined with the project study report, preliminary embodiment and historical information, etc., a simple quantitative evaluation. Government should risk the project's initial recognition, and pattern Ppp brief assessment.

Preparation phase: the preparation phase of the project, assuming the same output performance 
of the project, the public sector carefully test program comparison value is determined Ppp project model can reduce life-cycle costs of the project.

Procurement stage: in the procurement stage the government can play to encourage social capital initiative by the competition, optimize the life of the project design, operation and management of the corresponding programs. In the late procurement, review the terms of the contract as well as the focus of the government's responsibility to pay.

The implementation phase: Ppp project life cycle is longer, the larger the impact of environmental factors on the objective of the project implementation, the project should be based on the stage of the treaty, according to the external environment appropriate adjustments and changes in the pricing of risk transfer, to ensure that projects have a stable social capital earnings expectations. It should be noted at the same time strengthen the performance evaluation, prevent value of Ppp to be high.

Transition phase: This phase is the final phase of the project life cycle Ppp deal with project cost-effectiveness calculations, determination of social capital performance.

\section{References}

[1] Li Lin, Liu Zhihua, Zhang Kun Chang and other participants status Game Model Asymmetric PPP project risk allocation [J]. Systems Engineering - Theory \& Practice, 2013,33 (8): 1940-1948.

[2] Dua Ling, Yan Peng .PPP project contracting risk control framework study - based on trust and enhance maintain perspective [J] Wuhan University of Technology (Social Science Edition), 2013,26 (6): 880-886.

[3] YE Su, Wushu Xia, and other single Xueqin of PPP projects and cooperation in the interests of distribution [J] Science and Technology Progress and Policy, 2010,27 (19): 36-39.

[4] Anhui, dew Zheng, Zheng Zhuanjun Game PPP and other project cooperation surplus allocation under Incomplete Contract Perspective [J] Journal of Civil Engineering and Management, 2014, (2): 73-77. 